\title{
MTORC 1/2 Inhibitor LXI-15029
}

National Cancer Institute

\section{Source}

National Cancer Institute. mTORC 1/2 Inhibitor LXI-15029. NCI Thesaurus. Code C148528.

An orally bioavailable inhibitor of raptor-mammalian target of rapamycin (mTOR) complex 1 (mT OR complex 1; mT ORC1) and rictor-mT OR complex 2 (mT OR complex 2; mTORC2), with potential antineoplastic activity. Upon oral administration, mT ORC1/2 inhibitor LXI-15029 binds to the kinase domain of mT OR and inhibits both mTORC1 and mTORC2, in an ATP-competitive manner. This inhibits mTOR-mediated signaling and leads to both an induction of apoptosis and a decrease in the proliferation of mTORC1/2expressing tumor cells. mTOR is a serine/threonine kinase that is upregulated in certain tumor cell types. It plays an important role in the PI3K/Akt/mT OR signaling pathway, which is often deregulated in cancer cells and promotes cell growth, survival, and resistance to chemotherapy and radiotherapy. 